\title{
Measuring colour rivalry suppression in amblyopia
}

Timothy S Hofeldt, Albert J Hofeldt

\begin{abstract}
Aims-To determine if the colour rivalry suppression is an index of the visual impairment in amblyopia and if the stereopsis and fusion evaluator (SAFE) instrument is a reliable indicator of the difference in visual input from the two eyes.

Methods-To test the accuracy of the SAFE instrument for measuring the visual input from the two eyes, colour rivalry suppression was measured in six normal subjects. A test neutral density filter (NDF) was placed before one eye to induce a temporary relative afferent defect and the subject selected the NDF before the fellow eye to neutralise the test NDF. In a non-paediatric private practice, 24 consecutive patients diagnosed with unilateral amblyopia were tested with the SAFE. Of the 24 amblyopes, 14 qualified for the study because they were able to fuse images and had no comorbid disease. The relation between depth of colour rivalry suppression, stereoacuity, and interocular difference in $\log$ MAR acuity was analysed.
\end{abstract}

Results-In normal subjects, the SAFE instrument reversed temporary defects of 0.3 to $1.8 \mathrm{log}$ units to within $0.6 \mathrm{log}$ units. In amblyopes, the NDF to reverse colour rivalry suppression was positively related to interocular difference in $\log M A R$ acuity $(\beta=1.21, p<0.0001)$, and negatively related to stereoacuity $(\beta=-0.16, p=0.019)$. The interocular difference in $\log$ MAR acuity was negatively related to stereoacuity $(\beta=-0.13, p=0.009)$.

Conclusions-Colour rivalry suppression as measured with the SAFE was found to agree closely with the degree of visual acuity impairment in non-paediatric patients with amblyopia.

(Br f Ophthalmol 1999;83:1283-1286)

It has been recognised recently that a relative afferent defect can influence the fusion of rival colour images on the Worth 4 dot test. ${ }^{1}$ In diseases affecting optic nerve function, recovery of fusion on the Worth 4 dot test was obtained by applying a neutral density filter (NDF) in front of the eye without the defect. ${ }^{1}$ A significant positive association was identified between the NDF necessary to neutralise the relative afferent pupillary defect and the NDF necessary to reverse colour suppression. ${ }^{1}$ The relative afferent pupillary defect was not, however, a prerequisite for colour suppression as some subjects without a relative afferent pupil- lary defect required a NDF to obtain fusion on the Worth 4 dot test. ${ }^{1}$ To our knowledge, measurement of reversal of suppression of rival colour images in amblyopia has not been reported.

We tested a new instrument, the SAFE (Stereopsis and Fusion Evaluator, Gulden Ophthalmics, Elkins Park, PA, USA), which is a modified ViewMaster stereo viewer designed to simplify the detection and quantification of colour rivalry suppression. The device also assesses simultaneous perception of contour images, contour fusion, and stereopsis.

In this study we (1) determined the accuracy of the SAFE instrument to measure colour rivalry suppression in normal subjects, (2) measured colour rivalry suppression in amblyopes, and (3) related the depth of colour rivalry suppression in amblyopia to stereoacuity and interocular difference in visual acuity.

\section{Patients and methods}

A group of six normal subjects was tested to determine the precision of the SAFE instrument at quantifying an induced temporary unilateral afferent defect. This group consisted of volunteers having no knowledge of the vision concepts being studied and no abnormal finding on comprehensive eye examination. Each subject had $20 / 20$ or better corrected visual acuity in each eye and stereopsis of at least 150 seconds of arc (tested with the SAFE instrument). Age ranged from 23 to 61 years and the mean was 43 years.

The diagnosis of amblyopia was established by a history of unilateral reduced visual acuity since early life and visual acuity of $20 / 30$ or worse in the amblyopic eye. Visual acuity was tested with lines of letters at 20 feet; credit was given for the line if four of five Snellen letters were seen correctly. One examiner (TSH) performed the SAFE test and another examiner $(\mathrm{AJH})$ performed the ophthalmic examination and refraction. Neither examiner was aware of the findings of the other investigator until conclusion of testing.

Twenty four consecutive amblyopes seen in a non-paediatric private practice were examined. Their ages ranged from 10 to 79 years and the mean was 46 years. Ten amblyopes were excluded, eight because of comorbid disease (three with cataract, one with glaucoma, one with myopic degeneration, one with macular hole, one with uveitis, one with colour blindness), and two lacked simultaneous perception (one with exotropia, one with severe anisometropia amblyopia). The 14 amblyopes included in the study had no comorbid disease of the visual system and
Accepted for publication 28 May 1999 

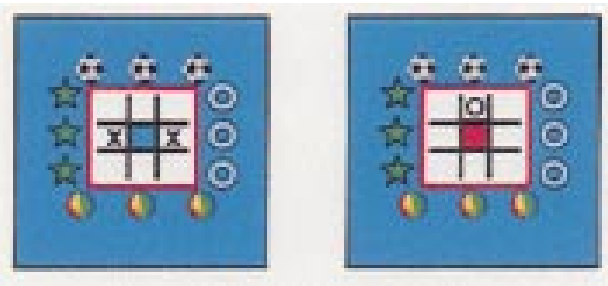

Figure 1 The stereo image pair viewed in the $S A F E$ instrument.

demonstrated simultaneous perception of contour images either at baseline or during the reversal process - that is, while dimming the image to the non-amblyopic eye. The aetiology of amblyopia was anisometropia in 12 patients and strabismus in two patients. One strabismic patient had accommodative esotropia during childhood and was orthophoric at testing and the other had 4 prism dioptres of esotropia. The Snellen visual acuity of all nonamblyopic eyes was $20 / 25$ or better. In the amblyopic eyes, vision ranged from $20 / 30$ to 20/200 (see Table 2).

The SAFE instrument is a lighted ViewMaster stereo viewer modified by mounting stereo images (Fig 1) within the viewer and substituting NDFs for the stereo images mounted in the ViewMaster reel. The fixation targets consist of four stereograms, a binocularly viewed grid in the shape of a tic tac toe (fusible, non-rivalrous), monocularly viewed two Xs and one $\mathrm{O}$ (non-fusible, non-rivalrous), and a coloured centre square (fusible, rivalrous) which is red for one eye and blue-green for the opposite eye. The stereograms subtend 1200 (beach balls), 600 (soccer balls), 300 (stars), and 150 (rings) seconds of arc. The coloured centre square subtends 1.7 degrees. With the SAFE instrument in the upright position, the right eye views a red centre square (hue 0, saturation 255, luminance 128, red 255, green 0, blue 0; PowerPoint program Microsoft Corporation, Redmond, WA, USA) and the left eye views a blue-green centre square (hue 137, saturation 255, luminance 120, red 0, green 191, blue 240). Kodak Wratten NDFs (Eastman Kodak Company, Rochester, NY, USA) increasing in $0.3 \log$ unit increments are mounted in the seven left ports of ViewMaster reel. Two reels are required to encompass the filter range from 0 to $3.0 \mathrm{log}$ units; one reel ranged from 0 to $1.8 \mathrm{log}$ units, the other from 1.2 to $3.0 \log$ units. By advancing the NDF reel, the difference in interocular brightness can be varied from 0 to $3.0 \mathrm{log}$ units. With the viewer in the position viewing the blue-green centre square, the patient advanced the NDF reel (dimming the eye

Table 1 Neutral density filters (log units) selected by normal subjects to match test filters

\begin{tabular}{|c|c|c|c|c|c|c|c|c|c|c|c|c|}
\hline \multirow{2}{*}{$\begin{array}{l}\text { Test } \\
\text { filter }\end{array}$} & \multicolumn{2}{|c|}{ Subject 1} & \multicolumn{2}{|c|}{ Subject 2} & \multicolumn{2}{|c|}{ Subject 3} & \multicolumn{2}{|c|}{ Subject 4} & \multicolumn{2}{|c|}{ Subject 5} & \multicolumn{2}{|c|}{ Subject 6} \\
\hline & $R$ & $L$ & $R$ & $L$ & $R$ & $L$ & $R$ & $L$ & $R$ & $L$ & $R$ & $L$ \\
\hline 0.3 & 0 & 0 & 0 & 0 & 0 & 0.6 & 0.6 & 0 & 0.9 & 0.6 & 0.6 & 0 \\
\hline 0.6 & 0 & 0.6 & 0.3 & 0.3 & 0.9 & 0.6 & 0.9 & 0.6 & 0.9 & 1.2 & 0.6 & 0.6 \\
\hline 0.9 & 0.6 & 0.9 & 0.6 & 0.9 & 1.2 & 1.2 & 1.5 & 0.9 & 1.2 & 1.2 & 1.2 & 0.6 \\
\hline 1.2 & 1.2 & 1.8 & 0.9 & 1.2 & 1.2 & 1.2 & 1.8 & 0.9 & 1.5 & 1.5 & 1.5 & 1.2 \\
\hline 1.5 & 1.5 & 1.8 & 1.2 & 1.5 & 1.8 & 1.8 & 2.1 & 1.5 & 1.5 & 2.1 & 1.8 & 1.5 \\
\hline 1.8 & 1.8 & 2.1 & 1.5 & 1.8 & 1.8 & 1.8 & 2.1 & 1.8 & 2.1 & 2.1 & 2.4 & 2.1 \\
\hline
\end{tabular}

without the relative afferent defect) until the colour of the centre square changed to red (end point). To verify the end point, the filter density was reduced to $0.3 \log$ units below NDF required for reversal of suppression (subthreshold NDF), and end point was repeated. The threshold for colour rivalry suppression is crossed a minimum of three times with this protocol. The end point was accepted when (1) the centre appeared red at baseline when the viewer was held in one position and blue-green in the other position, and (2) some blue-green was perceived with the subthreshold NDF over the eye without the relative afferent defect and end point was reached with a single advancement of the filter reel (0.3 log units).

In the neutralisation experiment, the test NDF was selected at random from a $0.3,0.6$, $0.9,1.2,1.5$, and $1.8 \log \mathrm{NDF}$ series and placed before the right ocular (the side with the red centre square) of the instrument. For each test NDF, the subjects were first tested with the SAFE instrument in the upright position and then in the upside down position.

For the amblyopes, the amblyopic eye was identified by the position of the SAFE instrument when the patient perceived the centre square as blue-green. If blue-green was perceived in the upright position, the right eye was the amblyopic eye; if blue-green was perceived in the upside down position, the left eye was the amblyopic eye.

In the neutralisation experiment, linear regression factors were used to evaluate how well the NDF selected by the subject agreed with the test NDF placed before the opposite eye. In the amblyopic series, linear regression factors were used to define the relation between (1) the interocular difference in $\log$ MAR acuity and the NDF necessary to reverse colour rivalry suppression, (2) stereoacuity and the NDF necessary to reverse colour rivalry suppression, and (3) the interocular difference in $\log$ MAR acuity and stereoacuity. For regression analysis, subjects were scored for their finest discernible stereoacuity: no stereopsis $=0 ; 1200$ seconds of arc $=$ $1 ; 600$ seconds of arc $=2 ; 300$ seconds of arc $=$ $3 ; 150$ seconds of arc $=4$. The regression coefficient, $\mathrm{p}$ values, and paired Student's $t$ test were calculated using SAS (SAS Institute Inc, Cary, NC, USA).

\section{Results}

In neutralising the test NDFs $(0.3-1.8 \log$ units) all end points were within $0.6 \log$ units (Table 1). Neutralisation and test NDF were of the same optical density in $32 \%$ ( 23 of 72 end points), $0.3 \log$ unit difference in $55 \%$ (40 of 72 end points), and $0.6 \log$ unit difference in $13 \%$ (nine of 72 end points). Regression analysis comparing the densities of the neutralisation NDFs with those of the test NDFs for each subject yielded $\beta$ values ranging from 0.79 to $1.32 ; \mathrm{p}$ values were less than or equal to 0.001 . The $\beta$ value was 1.13 and $p<0.0001$ for the group of 12 eyes. The $\beta$ value was 1.09 with $\mathrm{p}<0.0001$ for the right eyes, and 1.17 with $\mathrm{p}<0.0001$ for the left eyes. Paired Student's $t$ 
Table 2 Amblyopic patients

\begin{tabular}{|c|c|c|c|c|c|c|}
\hline Case & Type & Age & $\begin{array}{l}\text { Log } \\
\text { filter }\end{array}$ & $\begin{array}{l}\text { Stereo- } \\
\text { acuity } \\
\text { (arc s) }\end{array}$ & $\begin{array}{l}\text { Acuity in } \\
\text { amblyopic } \\
\text { eye } \\
\text { (Snellen) }\end{array}$ & $\begin{array}{l}\text { Acuity } \\
\text { difference } \\
\text { (log } M A R)\end{array}$ \\
\hline 1 & A & 10 & 1.2 & 150 & $20 / 30$ & 0.18 \\
\hline 2 & A & 19 & 1.2 & 150 & $20 / 30$ & 0.18 \\
\hline 3 & A & 79 & 1.2 & 150 & $20 / 30$ & 0.08 \\
\hline 4 & $\mathrm{~S}$ & 67 & 1.5 & 1200 & $20 / 40$ & 0.20 \\
\hline 5 & A & 18 & 1.5 & 0 & $20 / 80$ & 0.51 \\
\hline 6 & A & 49 & 1.5 & 0 & $20 / 40$ & 0.30 \\
\hline 7 & A & 52 & 1.5 & 1200 & $20 / 60$ & 0.48 \\
\hline 8 & S & 64 & 1.5 & 600 & $20 / 30$ & 0.08 \\
\hline 9 & A & 70 & 1.5 & 150 & $20 / 30$ & 0.08 \\
\hline 10 & A & 33 & 1.8 & 0 & $20 / 100$ & 0.60 \\
\hline 11 & A & 38 & 1.8 & 0 & $20 / 50$ & 0.40 \\
\hline 12 & A & 56 & 2.1 & 0 & $20 / 200$ & 0.90 \\
\hline 13 & A & 35 & 2.4 & 1200 & $20 / 200$ & 1.00 \\
\hline 14 & A & 64 & 2.7 & 0 & $20 / 200$ & 1.00 \\
\hline
\end{tabular}

Amblyopia type: $\mathrm{A}=$ anisometropic, $\mathrm{S}=$ strabismic.

test disclosed no significant difference between right and left eyes, $\mathrm{p}=0.613$.

For the amblyopes, the NDF necessary for reversal of colour rivalry suppression ranged from 1.2 to $2.7 \log$ units with a mean filter density of $1.6 \mathrm{log}$ units and a mode of $1.5 \mathrm{log}$ units (Table 2). Two subjects (nos 12 and 14) displayed suppression of contour images (monocular perception) at baseline, yet appreciated simultaneous perception and contour fusion after dimming the image to the nonamblyopic eye during reversal of colour rivalry suppression. For subject no 14, simultaneous perception, contour fusion, and reversal of colour rivalry suppression occurred concurrently with a NDF of $2.7 \mathrm{log}$ units. Subject no 12 experienced simultaneous perception and contour fusion at $1.8 \mathrm{log}$ units, and reversal of colour rivalry suppression at $2.1 \mathrm{log}$ units. During testing and upon reversal of colour rivalry suppression, stereoacuity did not improve above that demonstrated at baseline. Of the 14 amblyopes, eight (57\%) demonstrated stereopsis with the SAFE instrument. The finest discernible stereoacuity measured in seconds of arc was 150 for four patients, 600 for one patient, and 1200 for three patients.

Linear regression comparing the NDF necessary to reverse colour rivalry suppression and the $\log$ MAR acuity difference between all amblyopic and fellow, non-amblyopic eyes yielded a $\beta$ value of 1.21 with $p<0.0001$ (Fig 2). A significant negative relation was found

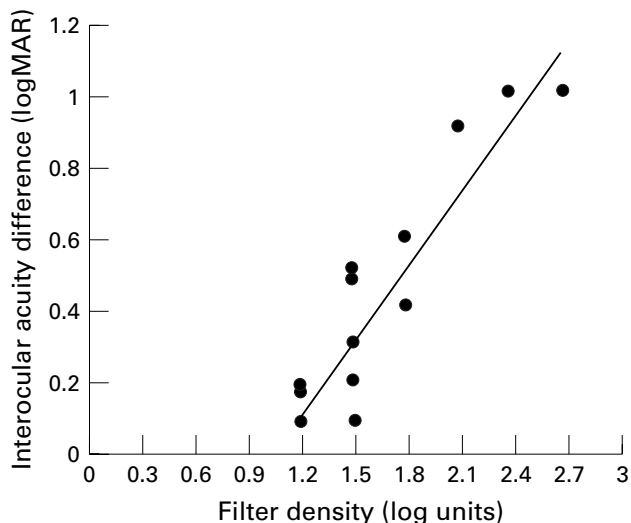

Figure 2 Relation between the NDF to reverse colour rivalry suppression and the depth of amblyopia in $\log M A R$ acuity. between the number of correctly seen stereo images and (1) the NDF necessary to reverse colour rivalry suppression ( $\beta$ value of -0.16 ; $\mathrm{p}=0.019$ ), and (2) the interocular difference in $\log$ MAR acuity $(\beta$ value of $-0.13 ; p=0.009)$.

\section{Discussion}

Stimulation of corresponding retinal areas with dissimilar (fusible, rivalrous) colours forms the basis of evaluating a relative afferent defect with the SAFE instrument. The centre square is equivalent to the white light of the Worth 4 dot test, the only one of the four Worth lights viewed binocularly. The reversal of colour rivalry suppression follows a sequence similar to that of fusion recovery with the Worth 4 dot test. ${ }^{1}$ Johnson reported an orderly progression from suppression to retinal rivalry to fusion and then to reverse suppression as NDFs of increasing optical density were placed over the eye without the relative afferent defect while viewing the 4 dot flashlight. ${ }^{1}$ We found the SAFE instrument a reliable indicator of difference in visual input from the right and left eyes in normal subjects viewing through unilateral test NDFs and in amblyopes. The amblyopic eye is identified when the amblyope perceives a blue-green centre square at baseline-that is, when the amblyopic eye is viewing the red image and the non-amblyopic eye is viewing the blue-green image. The depth of suppression is then measured by dimming the blue-green image seen by the non-amblyopic eye until rivalry is dominated by the red image viewed by the amblyopic eye. The reverse suppression threshold is verified by crossing the threshold twice more.

We found the accuracy of SAFE instrument to be within $0.6 \mathrm{log}$ units in neutralising experimentally induced temporary unilateral afferent defects; $87 \%$ of the end points were within $0.3 \log$ units and $100 \%$ were within 0.6 $\log$ units. Agreement between the test NDF and the neutralisation NDF was positively correlated for the subjects as a group $(\mathrm{p}<0.0001)$ and as individuals $(\mathrm{p}<0.001)$. The NDFs necessary to reverse test NDFs were very similar for the left and right eyes, $\beta$ values of 1.09 and 1.17 , respectively.

After demonstrating that the SAFE instrument can accurately quantify an induced temporary relative afferent defect, we used the instrument to quantify the amblyopic defect in 14 amblyopes. The minimum NDF necessary to reverse colour rivalry suppression in amblyopes was $1.2 \log$ units. Two of the amblyopes that lacked simultaneous perception of contour images at baseline, acquired contour fusion, and reversal of colour rivalry suppression by dimming the non-amblyopic eye. In all 14 amblyopes, stereoacuity did not show improvement from that displayed at baseline during the process of reversal of colour rivalry suppression; a similar finding was reported by Johnson ${ }^{1}$ in patients with optic nerve diseases.

We found three variables significantly interrelated in amblyopes, the NDF necessary to reverse colour rivalry suppression, the level of stereoacuity, and the logMAR acuity difference between the eyes. The most striking finding is 
the strong positive relation between the NDF required for reversal of colour rivalry suppression and the visual acuity impairment $(p<0.0001)$. Of lesser significance were $(1)$ the inverse relation between the level of stereoacuity and the visual acuity impairment $(p=0.009)$, and (2) the inverse relation between the NDF required for reversal of colour rivalry suppression and the level of stereoacuity $(\mathrm{p}=0.019)$.

Identified defects in amblyopia include (1) the relative afferent pupillary defect, ${ }^{2-5}$ (2) reduced resolution and contrast sensitivity, ${ }^{67}$ (3) spatial uncertainty, ${ }^{7}$ (4) a small difference in isoluminance points between the amblyopic and non-amblyopic eye, with the amblyopic eye possessing a relative defect for perception of red, ${ }^{8}(5)$ reduced stereo acuity, ${ }^{9}{ }^{10}$ (6) visual evoked potential (VEP) changes, ${ }^{311}$ (7) colour vision changes, ${ }^{3} 1213$ and (8) visual field defects. ${ }^{14}$

Using contrast sensitivity, investigators ${ }^{15}$ have studied two perceptual factors in amblyopia (1) the degree of amblyopia (difference in monocular contrast threshold between the two eyes) and (2) the depth of suppression (the amount by which the monocular contrast increment threshold for an eye was elevated by stimulation of the contralateral eye). They found the degree of amblyopia, as measured by contrast ratio, directly related to the interocular acuity ratio $(r=0.66$, calculated from their data) while the degree of suppression was inversely related to the interocular acuity ratio $(r=-0.69)$. Determination and subsequent analysis of SAFE scores makes no attempt to separate or differentiate the monocular defect from the confounding effect of suppression. The SAFE instrument measures the bulk deficit of the amblyopic eye relative to the fellow normal eye in the dichoptic condition, and presumably is affected by both the degree of amblyopia and depth of suppression.

The following case illustrates that the SAFE instrument can be valuable in excluding the diagnosis of amblyopia. A 44 year old patient presented with a history of having a weak left eye since childhood. She had been told by an ophthalmologist that she had amblyopia and the best corrected acuity was 20/60 in her left eye. She had never worn glasses or a patch as a child, nor used any correction at present. Despite uncorrected visual acuity of 20/30 in the right eye and 20/400 in the left eye, an end point of only $0.6 \mathrm{log}$ units was found in the left eye with the SAFE instrument. This degree of interocular difference is within the accuracy range of the instrument that we found in the normal group. From our data on amblyopes, colour rivalry suppression of about $1.5 \mathrm{log}$ units would be expected in amblyopia with vision loss of 20/60. Careful manifest refraction disclosed anisometropia and an absence of amblyopia. She was correctable to $20 / 20$ in each eye with plano -0.75 dioptres at 35 degrees for the right eye and +2.50 dioptres -6.00 dioptres at 150 degrees for the left eye. From our study, amblyopia of 20/30 or worse should be suspected when colour rivalry suppression measures at least 1.2 log units.

The SAFE instrument is a modified ViewMaster stereo viewer and has a fixed interpupillary distance of $65 \mathrm{~mm}$ with a tolerance of 62-68 $\mathrm{mm}$. Because the interpupillary distance of 5 year old children is about $50 \mathrm{~mm}$, the SAFE instrument is not appropriate for testing young children. This fact is of particular surprise considering the ViewMaster is marketed for children of 3 years and older. The strong relation we found between colour suppression and visual acuity impairment in amblyopia over age 10 years old raises an interesting question: would a test based on colour rivalry have the potential of screening for amblyopia in children?

The authors thank Paul Meier, $\mathrm{PhD}$, and Luis Mateo, $\mathrm{PhD}$, from the Department of Public Health, Columbia University, for performing the statistical analysis.

1 Johnson L. The relative afferent pupillary defect and a novel method of fusion recovery with the Worth 4-dot test. Arch Ophthalmol 1996;114:171-5.

2 Ikeda $\mathrm{H}$. Visual acuity, its development and amblyopia. $f R$ Soc Med 1980;73:546-55.

3 Portnoy JZ, Thompson HS, Lennarson L, et al. Pupillary defects in amblyopia. Am f Ophthalmol 1983;96:609-14.

4 Greenwald MJ, Folk ER. Afferent pupillary defects in amblyopia. F Pediatr Ophthalmol Strabismus 1983;20:63-7. 5 Firth AY. Pupillary responses in amblyopia. Br f Ophthalmol 1990;74:676-80.

6 Harrad RA, Hess RF. Binocular integration of contrast information in amblyopia. Vis Res 1992;32:2135-50.

7 Hess RF. Is amblyopia an impediment to binocular function? Eye 1996;10:245-9.

8 Mullen KT, Sankeralli MJ, Hess RF. Color and luminance vision in human amblyopia: shifts in isoluminance, contrast ensitivity losses, and positional deficits. Vis Res 1996;36: $645-53$

9 Campos E. Amblyopia. Surv Ophthalmol 1995;40:23-39.

0 McKee SP, Harrad RA. Fusional suppression in ormal and stereoanomalous observers. Vis Res 1993;33:1645-58.

11 Nawratzki I, Auerbach E, Rowe H. Amblyopia ex anopsia. Am f Ophthalmol 1966;61:430-5.

12 Pokorny J, Smith VC, Verriest G, et al. Congenital and acquired color vision defects. New York: Grune and Stratton, 1979:339-48.

13 Marre M, Marre E. Colour vision in squint amblyopia. Mod Probl Ophthalmol 1978;19:308-13.

14 Donahue SP, Wall M, Kutzko KE, et al. Automated perimetry in amblyopia: a generalized depression. $A m$ f Ophthalmol 1999;127:312-21

15 Holopigian K, Blake R, Greenwald MJ. Clinical suppression and amblyopia. Invest Ophthalmol Vis Sci 1988;29:444-51. 